\title{
An Evidence-Based Review On The Use Of Perampanel For The Treatment Of Focal-Onset Seizures In Pediatric Patients
}

This article was published in the following Dove Press journal:

Neuropsychiatric Disease and Treatment

\author{
Blandine Dozières- \\ Puyravel (D) ' \\ Stéphane Auvin (iD) ${ }^{1,2}$ \\ 'Service de Neurologie Pédiatrique et \\ des Maladies Métaboliques, Hôpital \\ Robert Debré, Paris, France; '2Université \\ Paris Diderot, Sorbonne Paris Cité, \\ INSERM UMR I|4I, Paris, France
}

\begin{abstract}
Perampanel, a non-competitive AMPA receptor antagonist, is a once-daily oral antiepileptic drug approved for the treatment of focal seizures and primary generalized tonicclonic seizures in children 12 years of age and over. We conducted a systematic review of the data on perampanel in children and adolescents with focal-onset seizures. We found 21 published papers on the pediatric use of perampanel for focal-onset seizures, including 9 papers on clinical trials and ancillary studies of these trials, of which 2 focused on pharmacokinetics, 1 was a metaanalysis, and 9 were real-life studies ( 1 was prospective). Perampanel, as an adjunctive treatment for uncontrolled focal-onset seizures, results in an improvement of seizure control with a generally good safety profile. Evidence-based data are currently available for children 12 years of age and over. Higher amounts of perampanel might be required in patients taking enzymeinducing antiepileptic drugs. The most common adverse events are somnolence, dizziness and behavioral adverse events. The latter seems more frequent in adolescents than in adults and in adolescents with preexisting behavioral comorbidities. Current studies did not identify any shortterm impact of perampanel on cognitive functions. Its long half-life allows once-daily administration. Real-life studies further established that a decrease of the dose frequently resolves adverse events, as demonstrated in pivotal studies. Pharmacokinetic and safety studies are still ongoing in the younger population, down to 4 years of age, in order to apply for approval in this age group.
\end{abstract}

Keywords: antiepileptic drugs, children, epilepsy, focal onset seizure, perampanel

\section{Introduction}

Perampanel is a selective, non-competitive antagonist of $\alpha$-amino-3-hydroxy-5-methyl4-isoxazolepropionic acid (AMPA)-type glutamate receptors at the postsynaptic level. Perampanel is the first non-competitive AMPA receptor antagonist anti-seizure agent. Perampanel is a once-daily oral antiepileptic drug approved for focal seizures and primary generalized tonic-clonic seizures for children 12 years of age and over in Europe. ${ }^{1,2}$ The approval in pediatric ages has been given for focal seizures down to the age of 4 years in October 2018, while the application has been submitted to the European Medicine Agency early in 2019. ${ }^{1}$

After oral administration, absorption is complete and rapid without significant first-pass metabolism. ${ }^{3}$ Under fasting conditions, the time taken to reach the maximum concentration $\left(T_{\max }\right)$ is 0.5 to $2.0 \mathrm{hrs}$. In clinical studies, half-life $\left(t_{1 / 2}\right)$ values ranged from 53 to $136 \mathrm{hrs}$ (average of $105 \mathrm{hrs}$ in 19 Phase I studies). ${ }^{3}$ The steady-state concentration is reached within 2 to 3 weeks. The area under the concentration-time
Correspondence: Stéphane Auvin Service de Neurologie Pédiatrique et des Maladies Métaboliques, CHU Hôpital

Robert Debrém, 48, Boulevard Sérurier,

Paris CEDEX 19- 75935, France

Tel +33 | 40035724

Fax +33 I 40034774

Email stephane.auvin@aphp.fr 
curve increases in a dose-proportional manner. ${ }^{3}$ Perampanel is mainly metabolized by CYP3A4 and CYP3A5. The clearance of perampanel is enhanced by enzyme-inducing antiepileptic drugs. ${ }^{3}$ But perampanel decreased oxcarbazepine clearance (by 26\%) increasing its plasma concentration (by $35 \%)^{3}$

Perampanel has first been approved by the FDA and the EMA in 2012 for focal-onset seizures with or without focal to bilateral tonic-clonic evolution. ${ }^{1,2}$ Initial approvals were given in adolescents above 12 years of age and in adults. Ancillary studies and real-life studies have been conducted since 2012 increasing our knowledge on the efficacy and safety of perampanel for epileptic disorders. In this article, we conducted a systematic review of the data on perampanel in children and adolescents for focal-onset seizures.

\section{Methods}

The systemic review has been done using the National Library of Medicine. Two searches have been realized with the following keywords: "perampanel AND children" and "perampanel AND adolescent". This has been conducted between February $10^{\text {th }}$ and February $15^{\text {th }}, 2019$. Only papers in English have been considered. The abstracts were first screened to identify all the papers on the administration of perampanel for focal-onset seizures. The authors conducted independently this bibliographic evaluation before sharing their results. The selection of the papers included in this review is reported in Figure 1.

Results are presented in three sections: data from randomized controlled trials, ancillary studies of the pivotal studies, and real-life studies.

\section{Results}

After analyzing the articles, we found 21 published papers on perampanel for the treatment of focal-onset seizures (Figure 1). The data are summarized below.

\section{Pivotal Studies}

Three Phase III pivotal studies were conducted to evaluate the efficacy and safety of perampanel in patients with drug-resistant epilepsy with focal-onset seizures (study 304: NCT00699972; 305: NCT00699582; 306: NCT00700310). ${ }^{4-7}$ These three studies were randomized, double-blind, placebo-controlled trials on perampanel use in addition to the antiepileptic drug regimen of the included patients ( 1 to 3 AEDs). Patients continued receiving their established concomitant AEDs without modification during the duration of the trial. These studies had a similar design with a 6-week observation pre-randomization phase, followed by a 19 -week, double-blind treatment phase divided into a titration phase of 6 weeks and a maintenance phase of 13 weeks. Perampanel was used from $2 \mathrm{mg} /$ day up to $12 \mathrm{mg} /$ day. Two studies evaluated perampanel $8 \mathrm{mg} /$ day and $12 \mathrm{mg} /$ day, ${ }^{4}$ while study 306 evaluated lower doses of $2 \mathrm{mg} /$ day, $4 \mathrm{mg}$ /day, and 8 $\mathrm{mg} /$ day. ${ }^{7}$ During the titration period, perampanel oral doses given before bedtime were increased by $2 \mathrm{mg} /$ day/ week. One downtitration of $2 \mathrm{mg}$ was permitted in case of adverse events.

The patients included in these three studies rolled over into an extension study starting by a blinded conversion period (NCT00735397). This open-label study was planned for 256 weeks. The aim of this study was to evaluate the maintenance of the efficacy, as well as the safety of perampanel at the maximum tolerated dose. The design allowed dose adjustment or discontinuation of the concomitant AED.

Rosenfeld et al reported the data of adolescent patients from the three pivotal RCT (studies 304, 305, or 306). ${ }^{6}$ Pooled data of 143 adolescents of the 1480 patients from the core studies showed a median percent decrease in seizure frequency of $34.8 \%$ for $8 \mathrm{mg} /$ day of perampanel and $35.6 \%$ for $12 \mathrm{mg} /$ day of perampanel, while the median

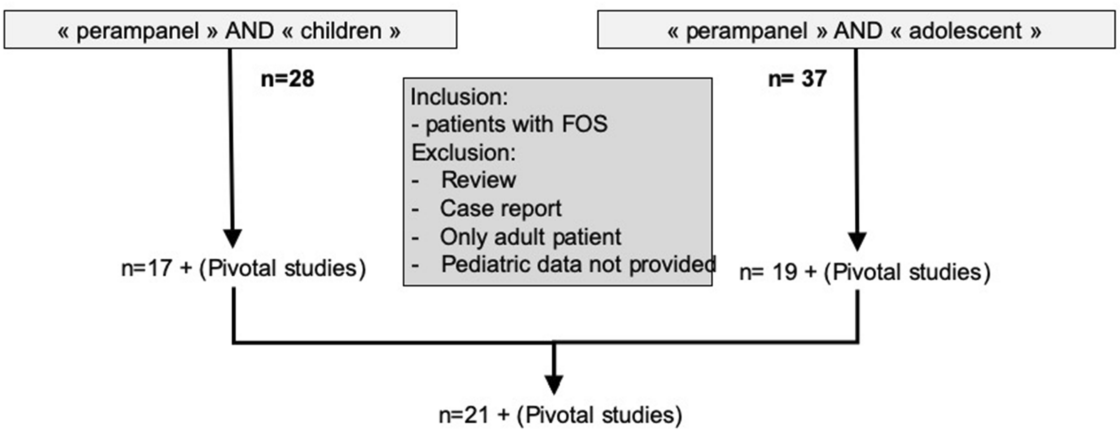

Figure I Graphic representation of the bibliographic research on perampanel use in children with uncontrolled focal-onset seizures. 
percent decrease in seizure frequency was $18 \%$ in the placebo group. ${ }^{6}$ The pooled analysis reported $22.2 \%$ of responder rate for placebo, $40.9 \%$ for $8 \mathrm{mg} /$ day of perampanel, and $45 \%$ for $12 \mathrm{mg}$ /day of perampanel (Table 1$)^{6}{ }^{6}$ When patients were under AED inducers (carbamazepine, oxcarbazepine, or phenytoin), a smaller median percent reduction in seizure frequency was observed in comparison to adolescents receiving concomitant AED noninducers (Table 1).

In the extension phase, 122 adolescents were analyzed with the intent-to-treat dataset, and 112 were included in the perampanel safety analysis. During the first 52 weeks of the open-label maintenance period, responder rates ranged from $27.3 \%$ to $60.0 \%$ in adolescents randomized to placebo during the core studies who switched to perampanel during the extension phase and from $40.9 \%$ to $54.8 \%$ in adolescents receiving perampanel throughout the study. ${ }^{6}$ During the double-blind phase, the most common adverse events defined by an occurrence of $>5 \%$ in perampaneltreated patients and $\geq 2$ times more often than with placebo were dizziness $(20.4 \%)$, somnolence $(15.3 \%)$, aggression $(8.2 \%)$, decrease of appetite $(6.1 \%)$, and rhinitis $(5.1 \%)$. Aggression was more frequent in the adolescent population than in the overall population, in particular with a higher dose. ${ }^{6}$ During the extension phase, the psychiatric and behavioral adverse events were aggression (18.2\%), insomnia (6.6\%), abnormal behavior (4.1\%) and anxiety $(4.1 \%){ }^{6}$

Another multicenter, randomized, double-blind, placebo-controlled trial involving adolescents aged $\geq 12$ years and adults with refractory focal-onset seizures further established the efficacy of perampanel (NCT01618695). ${ }^{8}$ Seven hundred and ten patients were randomized. This study found similar efficacy and safety profile than the above studies. The paper did not report the detailed data on the adolescent population. ${ }^{8}$

\section{Additional Randomized Controlled Trials Study 235 (NCTOI I6I524)}

A dedicated Phase II study has been conducted in adolescents with uncontrolled focal-onset seizures to evaluate the effect of perampanel on cognition, growth, safety, tolerability, and pharmacokinetics (study 235 (NCT01161524)). ${ }^{9}$ The design included 1 week of pre-randomization, followed by 6 weeks of titration, and finally a 19-week maintenance period. The objective of the study was to explore the relationship between perampanel exposure and the cognition assessed by CDR (Cognitive Drug Research) score (baseline versus Week 19). Seizure frequency, pharmacokinetics and safety, including behavior, were also evaluated. The study included 78 patients in the perampanel group and 46 in the placebo group, with a median age of 14 years (weight range from 28 to $116 \mathrm{~kg}$ with a median at $51.7 \mathrm{~kg}$ ). ${ }^{9}$

Pharmacokinetic parameters in the adolescent population were consistent with data in adults acquired during pivotal studies, suggesting the absence of age difference in pharmacokinetic dose between adults and adolescents aged 12 years and over. Moreover, dose linearity (ie, plasma concentration) increased in proportion to perampanel dose. This was similar in patients taking inducer and noninducer concomitant AED. ${ }^{9}$

The study was not sufficiently powered to evaluate the efficacy of perampanel versus placebo. The observed median percent change in seizure frequency from baseline per 28 days was $24 \%$ in the placebo group $(n=46)$ and $58 \%$ in the perampanel group $(\mathrm{n}=83)(\mathrm{p}=0.079$ but not powered to evaluate efficacy). The $50 \%$ responder rate was $37.0 \%(\mathrm{n}=17)$ in the placebo group versus $59.0 \%$ $(\mathrm{n}=49)$ in the perampanel group $(\mathrm{p}=0.0144$ but not powered to evaluate efficacy).

Another paper reported the details of the cognitive data of study $235 .{ }^{10}$ This study has used the CDR (cognitive drug research) system, which is a set of automated validated tests

Table I Efficacy Data Of Perampanel Based On The Pooled Data Of I43 Adolescents From The I480 Patients From The Core Studies $^{4-7}$

\begin{tabular}{|c|c|c|c|c|}
\hline & & Placebo & 8MG & I2MG \\
\hline Median percent decrease in Sz frequency & $\begin{array}{l}\text { FOS } \\
\text { with inducers } \\
\text { without inducers } \\
\text { Focal to bilateral TC }\end{array}$ & $\begin{array}{l}18 \% \\
15.4 \% \\
54.6 \% \\
40.3 \%\end{array}$ & $\begin{array}{l}34.8 \% \\
31.6 \% \\
52.7 \% \\
39.2 \%\end{array}$ & $\begin{array}{l}35.6 \% \\
26.8 \% \\
18 \% \\
4.4 \%\end{array}$ \\
\hline $\begin{array}{l}\text { Responders } \\
>50 \% \text { Sz reduction }\end{array}$ & FOS & $22.2 \%$ & $40.9 \%$ & $45 \%$ \\
\hline
\end{tabular}

Abbreviations: FOS, focal-onset seizure; Sz, seizure; TC, tonic-clonic. 
of cognitive function available in many languages. The CDR system has five core domains: 1) power of attention; 2) continuity of attention; 3) quality of episodic memory; 4) quality of working memory; and 5) speed of memory. Prior to dosing, it is important to note that epilepsy patients showed impairments on the CDR system in the power of attention domain as compared to healthy controls. In study 235, no difference was found between the perampanel group $(\mathrm{n}=79)$ and the placebo group $(\mathrm{n}=44) .{ }^{10}$ Precisely, perampanel resulted in a non-significant mean difference compared with placebo $(-2.2(95 \% \mathrm{CI}-5.2$ to $0.8) ; \mathrm{p}=0.145)$. Small differences between perampanel and placebo groups were found in few CDR system core domains. This resulted in an improvement in "quality of episodic memory" (perampanel: 3.0 vs placebo: -1.2 ; $\mathrm{p}=0.012$ ) and a worsening in 2 domains, "continuity of attention" (perampanel: -3.3 vs placebo: $1.6 ; p=0.013$ ) and "speed of memory" (perampanel: 0.3 vs placebo: 7.0; $\mathrm{p}=0.032$ ). Adverse events were reported with perampanel: dizziness in $30.6 \%$ and somnolence in $15.3 \%$.

Lagae et al reported on behavioral assessment in study 235 using the Child Behavior Checklist (CBCL) before and after 19 weeks in the studied groups (perampanel vs placebo). ${ }^{11}$ Sixteen items of the CBCL were scored for each adolescent and then grouped into three subscores for activities, social realms and school. The summation of the 16 items was used as a total competence score. No difference was found between the groups from baseline to the end of the study. The mean change in the CBCL total competence score after adjustment (region and age) was $0.0 \pm 3.54$ for perampanel $(\mathrm{n}=79)$ and $+0.2 \pm 3.52$ for placebo $(n=44)(p=0.619) .{ }^{11}$ No correlation was found between CBCL subscale scores and reduction in seizure frequency regardless of the group. ${ }^{11}$

An extension study was offered to the patients who completed the double-blind phase. ${ }^{12}$ The patients receiving the placebo in the first part of study 235 had a conversion period of 6 weeks. The open-label extension of 52 weeks was opened to patients who did not have access to perampanel (commercially not available or denied access program). One hundred and fourteen patients were included in the extension phase. A total of 24 patients (21.1\%) discontinued perampanel during the extension phase, with the most common reason for discontinuation being adverse events (thirteen patients received $10-12 \mathrm{mg} /$ day, ten received $6-8 \mathrm{mg} /$ day, and one received $4 \mathrm{mg}$ /day of perampanel). ${ }^{12}$ Using the CDR system to assess cognition at 52 weeks, the mean (SD) change in global cognition T-score was -1.0 (9.9) from baseline to the end of treatment; this change was not statistically significant $(p=0.96)$ and not considered to be clinically relevant. ${ }^{12}$ The extension phase also assessed the effect of perampanel on growth. No clinically relevant changes were observed in weight, height, or Tanner stage. ${ }^{12}$ Most of the common adverse events (ie, dizziness, somnolence, aggression, irritability, and fatigue) occurred during the first phase of the extension study (Weeks 1-13) and decreased in frequency in the second part of the extension phase (Weeks $40-52){ }^{12}$

\section{Ancillary Studies Or Pooled Analysis Of The Randomized Controlled Trials Data}

In addition to the randomized controlled trial data, our review identified 2 pharmacological studies, 1 pooled analysis on psychiatric side effects of perampanel, and an ancillary study on patients' risk of falling.

A pharmacokinetic/pharmacodynamic analysis of perampanel in patients with focal-onset seizure was available. ${ }^{13}$ Perampanel plasma concentrations during maintenance of the patients included in the pivotal studies, in study 335 , and in study 235 , were used for a pooled analysis. Pooled perampanel pharmacokinetic data were best described by a one-compartment disposition model with linear elimination parameterized for apparent clearance and volume of distribution (VD). Without co-administration of AED inducers, the apparent clearance is similar in the total population $(0.668 \mathrm{~L} / \mathrm{hr})$ and the adolescent population $(0.682 \mathrm{~L} / \mathrm{hr}) .{ }^{13}$ The apparent clearance of perampanel is increased in both populations with carbamazepine, oxcarbazepine/phenytoin, and topiramate/phenobarbital. ${ }^{13}$ In the pharmacokinetic/pharmacodynamic model, perampanel administration reduced the frequency of focal-onset seizures in relation to an increase in perampanel exposure, but independently of time. ${ }^{13}$ No intrinsic or extrinsic factors significantly affected the probability of response. ${ }^{13}$

A second study on pharmacokinetics was found in our literature review. It was a multicenter, open, prospective study on the effects of antiepileptic drugs on the steady-state plasma concentration of perampanel in epilepsy patients. ${ }^{14}$ Patients aged 12 years and over treated with a stable dose of perampanel for at least 1 month and stable concomitant antiepileptic drugs for the last 3 weeks were included. Blood samples were collected approximatively $12 \mathrm{hrs}$ after perampanel intake at bedtime to evaluate the perampanel plasma concentration to weight-adjusted daily dose ratio as the main primary outcome $\left(\mathrm{C} / \mathrm{D} ;[\mu \mathrm{g} / \mathrm{mL}] / \mathrm{mg} \mathrm{kg}^{-1} \mathrm{~d}^{-1}\right){ }^{14}$ This study 
reported the data from 114 blood samples from 79 patients (53\% female) with a mean age of $33 \pm 13$ years with a body weight of $68 \pm 21 \mathrm{~kg}$. The mean amount of perampanel prescribed was $6.3 \pm 2.3 \mathrm{mg} / \mathrm{day}$. Age and sex did not significantly affect perampanel C/D. The combination of perampanel with enzyme inducers significantly reduced perampanel $\mathrm{C} / \mathrm{D}$. The mean values of $\mathrm{C} / \mathrm{D}$ were similar in patients treated with carbamazepine, phenytoin, or oxcarbazepine. ${ }^{14}$

As mentioned above, psychiatric and behavioral side effects have been identified in epilepsy patients treated with perampanel. ${ }^{6}$ Ettinger et al analyzed the full perampanel safety database showing that psychiatric adverse events were associated with the use of perampanel. ${ }^{15}$ This included multiple Phase I, II, and III clinical studies for epilepsy and non-epilepsy patients and Phase I volunteer populations. ${ }^{15}$ The risk of anger or aggression was not increased with perampanel in non-epilepsy patients, while a dose-related increase of psychiatric adverse events was observed in epilepsy patients. Analyzing the results of a questionnaire, the authors observed that the incidence of hostility/aggression was higher in perampanel-treated epilepsy patients compared to placebo (perampanel 3.0 vs placebo $0.7 \%$ with a narrow definition and $11.8 \%$ vs $5.7 \%$, respectively, for a broader definition). These types of adverse events were manageable with dose adjustment or discontinuation in few cases. ${ }^{15}$ An analysis of the adolescent population (12-18 years, $\mathrm{n}=143)$ in comparison to the adult population $(n=1309)$ suggested that psychiatric adverse events are more frequent in adolescents. The most common psychiatric adverse event was aggression, seen in $8.2 \%$ of patients (none in the placebo group). ${ }^{15}$

Several side effects commonly seen with antiepileptic drugs such as drowsiness, dizziness, fatigue, imbalance, or irritability might increase the risk of falling in epilepsy patients. A pooled analysis of 3 pivotal studies on focalonset seizures (studies 304, 305 and 306) (-7 $^{4-7}$ focused on this issue showing that perampanel exposure in patients with focal-onset seizures is associated with falls, which are associated with increased doses. ${ }^{16}$ In adolescents (12-18 years), falls were more frequently reported as a treatmentemergent adverse event in the perampanel-treated group (3.1\%) than in the placebo group (2.2\%). The risk of falling was generally higher in the adult population, including a further risk when treated with perampanel (perampanel $4.9 \%$ vs placebo $3.6 \%$ ). Pharmacokinetic/ pharmacodynamic investigations suggested that higher plasma perampanel concentrations increase the probability of gait disturbance and falls. ${ }^{16}$

\section{Meta-Analysis}

Using a network meta-analysis, Rosati et al conducted comparisons across pediatric randomized control trials based on a common comparator (placebo or standard of care). When 2 different types of intervention needed to be compared without any available study, indirect evidence was obtained by studying each intervention versus a common comparator. Direct comparisons were evaluated by random-effect pairwise meta-analysis within a frequentist approach. Then, treatment effect was assessed using odds ratios (OR) and their 95\% confidence intervals (CI). For mixed-treatment comparison, a random-effects NMA within a Bayesian framework has been performed. Then, the treatment effects were assessed using OR and their $95 \%$ credible intervals ((CrI) the Bayesian equivalent to CI). ${ }^{17}$ After the selection of 46 RCT representing 5652 individuals randomized to $22 \mathrm{AED}$ or placebo, the network analysis for focal-onset seizures consisted of five studies for newly diagnosed epilepsy and 9 studies for drug-resistant epilepsy. Data on perampanel were only available for drug-resistant focal-onset seizure epilepsy, showing that perampanel (OR $=2.5,95 \% \mathrm{CrI}=1.1-5.8)$ was more effective compared to placebo. In the same analysis, it was also found that levetiracetam was more effective compared to placebo $(\mathrm{OR}=3.3,95 \% \mathrm{CrI}=1.3-7.6) .{ }^{17}$

\section{Real-Life Studies}

Nine real-life studies have been published ${ }^{18-26}$ (Table 2). Eight are retrospective studies, one is a prospective study, ${ }^{25}$ and one is both prospective and retrospective. ${ }^{23}$ These studies reported a total of 407 children and adolescents treated with perampanel, including 255 patients with focal-onset seizures. The efficacy expressed by responders (decrease of at least $50 \%$ of seizure frequency compared to the baseline) varied from $12 \%$ to $62 \%$ for all patients. The efficacy data for focal-onset seizures were available in 3 of the nine 9 real-life studies, with a responder rate of $33 \%$ in the study published by Biro et al, while the responder rate was $19 \%$ at 6 months of treatment and $24 \%$ at 12 months of treatment in the study published by Swiderska et al. ${ }^{23}$ The adverse event rate varied from $13 \%$ to $70 \%$. The most frequent adverse events were behavioral adverse events, fatigue/somnolence and dizziness (Table 2). Some reports suggested that pretreatment behavioral comorbidity was a risk factor for the occurrence of behavioral adverse events, while the prescription of perampanel in association with levetiracetam did not seem to increase this risk (Table 2). Four studies mentioned that dose adjustment or discontinuation of perampanel leads to 


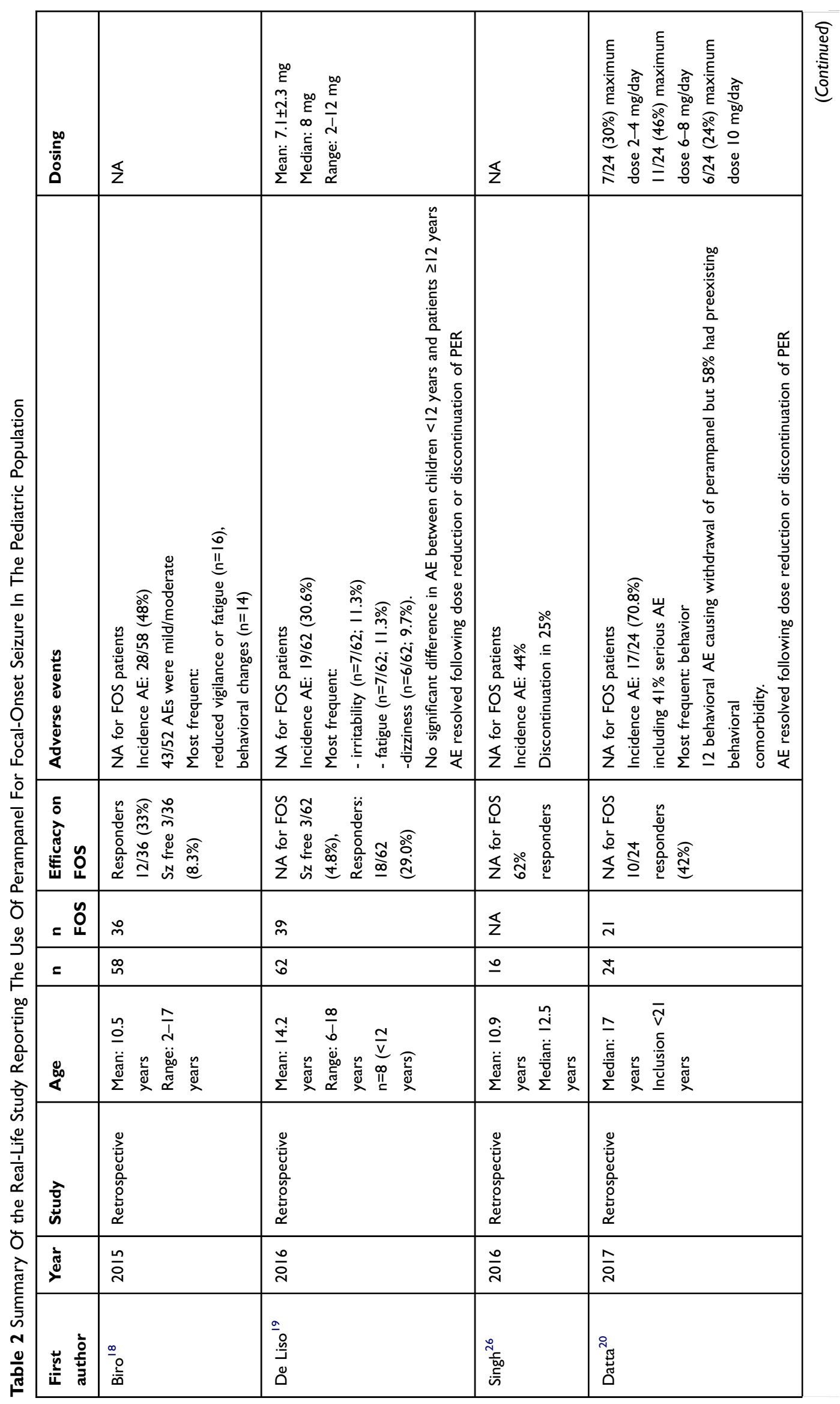




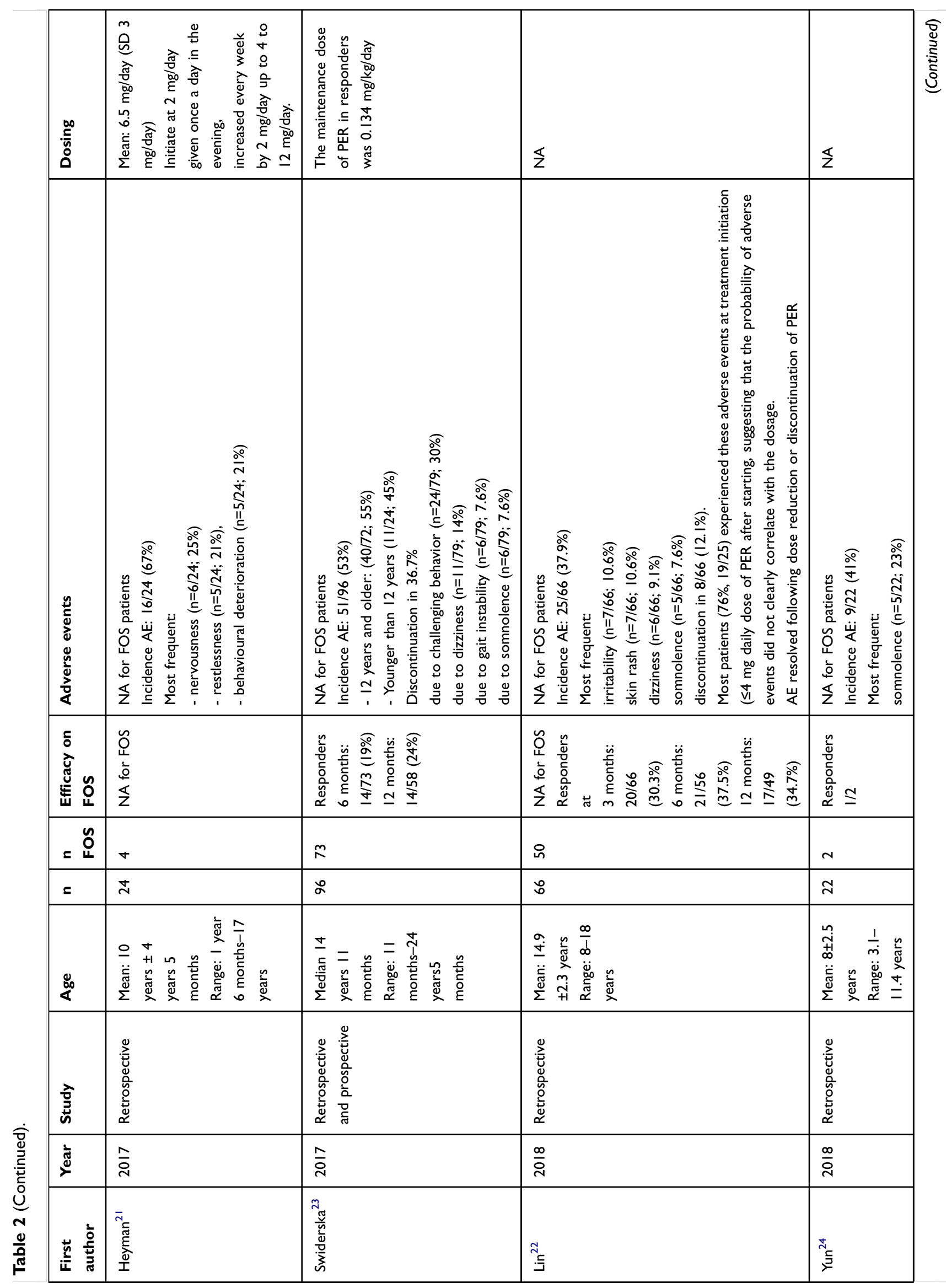




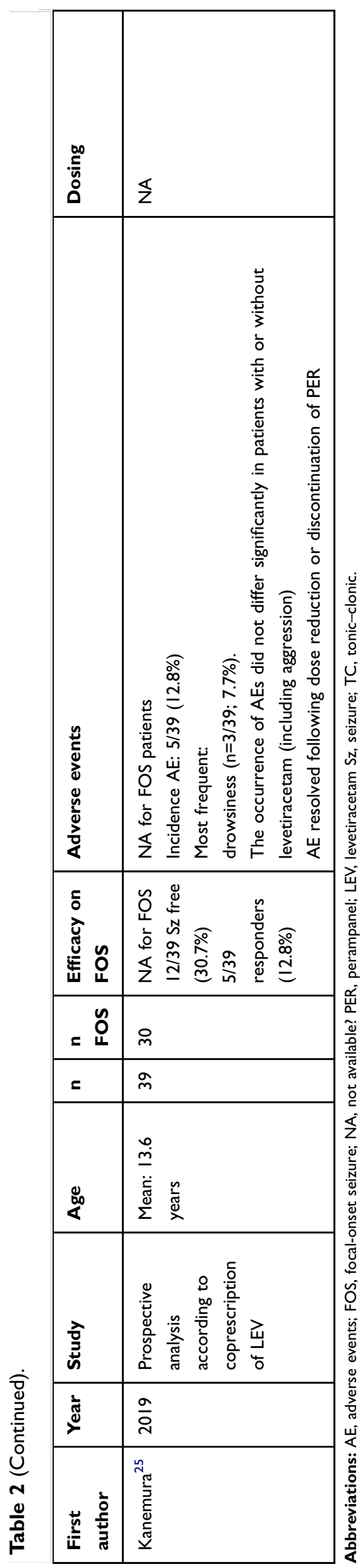

the disappearance of adverse events in most of the cases. Limited data on doses and titration were available. A titration of $2 \mathrm{mg}$ per day every week or every 2 weeks was most of the time used (Table 2).

\section{Conclusion}

As described above, adjunctive treatment with perampanel results in improved seizure control and is generally welltolerated in children aged 12 years and over with inadequately focal-onset seizure control. Notably, a prospective pharmacological study showed no evidence for clinically short-term effects of perampanel on overall cognitive function and behavior in adolescents. If a patient is treated by an enzyme-inducing antiepileptic drug, a higher dose of perampanel may be required. Despite a new mechanism of action, the efficacy of perampanel for focal-onset seizures does not seem really different compared to other available antiepileptic drugs. The adverse events are marked by behavioral adverse events, which are more frequent in adolescents than in adults.

The physician's decision on the choice of an antiepileptic drug for a child with focal-onset seizures is largely dependent on the patient characteristics and on literature about efficacy and safety profiles of these various drugs. Evidence-based guidelines are limited due to the absence of head-to-head comparison of antiepileptic drugs. The pharmacological profile of the compound and the adverse event profile are also taken into consideration. For perampanel, the once-daily administration and the long half-life represent two advantages for its use in the adolescent population. The low level of interaction with other drugs is also a positive point for female patients that might take oral contraceptives (estrogen-progesterone). The use of perampanel should probably be more cautious when a history of behavior comorbidity already exists in children and adolescents.

Drug development of perampanel is still ongoing in the pediatric population. Further studies should evaluate if there is any long-term effect of perampanel on cognition, growth and development in adolescents. An open-label study is currently ongoing on the evaluation of safety and tolerability of a perampanel oral suspension as adjunctive treatment (ages from 4 to 12 years) for children with inadequately controlled partial-onset seizures or primary generalized tonic-clonic seizures (NCT02849626). With the recent regulatory evolution on extrapolability of the data on the efficacy on adults, this study will probably lead 
soon to an application for drug approval for uncontrolled focal-onset seizures from 4 to 12 years in Europe.

\section{Acknowledgment}

The authors thank Sophie Höhn, $\mathrm{PhD}$, for her help in the preparation of the manuscript.

\section{Disclosure}

Dr Blandine Dozieres-Puyravel receives salary support from Assistance Publique - Hôpitaux de Paris. She is a coinvestigator on research grants awarded to Robert-Debré University Hospital from EISAI, UCB and Zogenix; she has received travel expenses or consulting fee from UCB, Biomarin, Novartis, EISAI, Advicenne, Vitaflo. Professor Stéphane Auvin receives salary support from Université de Paris and Assistance Publique - Hôpitaux de Paris. Stéphane Auvin is Associate Editor for Epilepsia. He is an investigator on research grants awarded to Robert-Debré University Hospital from Advicenne Pharma, EISAI, UCB Zogenix; he has received travel expenses or consulting fee from Arvelle, Advicenne Pharma, Biocodex, Biomarin, Eisai, GW Pharma, Novartis, Nutricia, Shire, UCB Pharma, Vitaflo, Zogenix. The authors report no other conflicts of interest in this work.

\section{References}

1. Available from: https://www.fycompa.com/-/media/Files/Fycompa/ Fycompa_Prescribing_Information.pdf. Accessed September 09, 2019.

2. EMA Perampanel approval. https://www.ema.europa.eu/en/medicines/ human/EPAR/fycompa\#authorisation-details-section. Accessed September 09, 2019.

3. Satlin A, Kramer LD, Laurenza A. Development of perampanel in epilepsy. Acta Neurol Scand. 2013;127:3-8. doi:10.1111/ane.2013. 127.issue-s197

4. French JA, Krauss GL, Biton V, et al. Adjunctive perampanel for refractory partial-onset seizures Randomized phase III study 304. Neurology. 2012;79 (6):589-596. doi:10.1212/WNL.0b013e3182635735

5. French JA, Krauss GL, Steinhoff BJ, et al. Evaluation of adjunctive perampanel in patients with refractory partial-onset seizures: results of randomized global phase III study 305. Epilepsia. 2013;54(1):117125. doi:10.1111/j.1528-1167.2012.03638.x

6. Rosenfeld W, Conry J, Lagae L, et al. Efficacy and safety of perampanel in adolescent patients with drug-resistant partial seizures in three double-blind, placebo-controlled, phase III randomized clinical studies and a combined extension study. Eur J Paediatr Neurol. 2015;19 (4):435-445. doi:10.1016/j.ejpn.2015.02.008

7. Krauss GL, Serratosa JM, Villanueva V, et al. Randomized phase III study 306 Adjunctive perampanel for refractory partial-onset seizures. Neurology. 2012;78(18):1408-1415. doi:10.1212/WNL.0b013e318254 $473 \mathrm{a}$

8. Nishida T, Lee SK, Inoue Y, Saeki K, Ishikawa K, Kaneko S. Adjunctive perampanel in partial-onset seizures: Asia-Pacific, randomized phase III study. Acta Neurol Scand. 2018;137(4):392-399. doi:10.1111/ane.12883
9. Villanueva V, Majid O, Nabangchang C, et al. Pharmacokinetics, exposure-cognition, and exposure-efficacy relationships of perampanel in adolescents with in adequately controlled partial-onset seizures. Epilepsy Res. 2016;127:126-134. doi:10.1016/j.eplepsyres.2016.08.025

10. Meador KJ, Yang HC, Pina-Garza JE, Laurenza A, Kumar D, Wesnes KA. Cognitive effects of adjunctive perampanel for partial-onset seizures: a randomized trial. Epilepsia. 2016;57(2):243-251. doi:10.1111/epi.13279

11. Lagae L, Villanueva V, Meador KJ, et al. Adjunctive perampanel in adolescents with inadequately controlled partial-onset seizures: a randomized study evaluating behavior, efficacy, and safety. Epilepsia. 2016;57(7):1120-1129. doi:10.1111/epi.13417

12. Pina-Garza JE, Lagae L, Villanueva V, et al. Long-term effects of adjunctive perampanel on cognition in adolescents with partial seizures. Epilepsy Behav. 2018;83:50-58. doi:10.1016/j.yebeh.2018.03.029

13. Takenaka O, Ferry J, Saeki K, Laurenza A. Pharmacokinetic/pharmacodynamic analysis of adjunctive perampanel in subjects with partial-onset seizures. Acta Neurol Scand. 2018;137(4):400-408. doi:10.1111/ane.12874

14. Contin M, Bisulli F, Santucci M, et al. Effect of valproic acid on perampanel pharmacokinetics in patients with epilepsy. Epilepsia. 2018;59(7):E103-E8. doi:10.1111/epi.14446

15. Ettinger AB, LoPresti A, Yang HC, et al. Psychiatric and behavioral adverse events in randomized clinical studies of the noncompetitive AMPA receptor antagonist perampanel. Epilepsia. 2015;56(8):12521263. doi:10.1111/epi.13054

16. Leppik IE, Yang HC, Williams B, et al. Analysis of falls in patients with epilepsy enrolled in the perampanel phase III randomized doubleblind studies. Epilepsia. 2017;58(1):51-59. doi:10.1111/epi.13600

17. Rosati A, Ilvento L, Lucenteforte E, et al. Comparative efficacy of antiepileptic drugs in children and adolescents: a network meta-analysis. Epilepsia. 2018;59(2):297-314. doi:10.1111/epi.13981

18. Biro A, Stephani U, Tarallo T, et al. Effectiveness and tolerability of perampanel in children and adolescents with refractory epilepsies: first experiences. Neuropediatrics. 2015;46(2):110-+. doi:10.1055/s0035-1546276

19. De Liso P, Vigevano F, Specchio N, et al. Effectiveness and tolerability of perampanel in children and adolescents with refractory epilepsies an Italian observational multicenter study. Epilepsy Res. 2016;127:93-100. doi:10.1016/j.eplepsyres.2016.08.021

20. Datta AN, Xu Q, Sachedina S, Boelman C, Huh L, Connolly MB. Clinical experience with perampanel for refractory pediatric epilepsy in One Canadian Center. J Child Neurol. 2017;32(9):834-839. doi:10.1177/0883073817709195

21. Heyman E, Lahat E, Levin N, et al. Tolerability and efficacy of perampanel in children with refractory epilepsy. Dev Med Child Neurol. 2017;59(4):441-444. doi:10.1111/dmen.13362

22. Lin KL, Lin JJ, Chou ML, et al. Efficacy and tolerability of perampanel in children and adolescents with pharmacoresistant epilepsy: the first real-world evaluation in Asian pediatric neurology clinics. Epilepsy Behav. 2018;85:188-194. doi:10.1016/j.yebeh.2018.06.033

23. Swiderska N, Tan HJ, Rajai A, Silwal A, Desurkar A, Martland T. Effectiveness and tolerability of Perampanel in children, adolescents and young adults with refractory epilepsy: a UK national multicentre study. Seizure. 2017;52:63-70. doi:10.1016/j.seizure.2017.08.014

24. Yun Y, Kim D, Lee YJ, Kwon S, Hwang SK. Efficacy and tolerability of adjunctive perampanel treatment in children under 12 years of age with refractory epilepsy. Korean J Pediatr. 2018;62:269-273.

25. Kanemura H, Sano F, Aihara M. Usefulness of perampanel with concomitant levetiracetam for patients with drug-resistant epilepsy. Eur $J$ Paediatr Neurol. 2019;23(1):197-203. doi:10.1016/j.ejpn.2018.10.004

26. Singh K, Shah YD, Luciano D, Friedman D, Devinsky O, Kothare SV. Safety and efficacy of perampanel in children and adults with various epilepsy syndromes: a single-center postmarketing study. Epilepsy Behav. 2016;61:41-45. doi:10.1016/j.yebeh.2016.05.007 


\section{Publish your work in this journal}

Neuropsychiatric Disease and Treatment is an international, peerreviewed journal of clinical therapeutics and pharmacology focusing on concise rapid reporting of clinical or pre-clinical studies on a range of neuropsychiatric and neurological disorders. This journal is indexed on PubMed Central, the 'PsycINFO' database and CAS, and is the official journal of The International Neuropsychiatric Association (INA). The manuscript management system is completely online and includes a very quick and fair peer-review system, which is all easy to use. Visit http://www.dovepress.com/testimonials.php to read real quotes from published authors. 